\title{
Evaluation of the Sensory Organization Test to differentiate non-fallers from single- and multi-fallers
}

\author{
Katarzyna Pierchała ${ }^{\mathrm{A}-\mathrm{F}}$, Magdalena Lachowska ${ }^{\mathrm{C}}{ }^{-}$, Jarosław Wysocki ${ }^{\mathrm{D}, \mathrm{E}}$, Krzysztof Morawski, ${ }^{\mathrm{E}, \mathrm{F}}$, Kazimierz Niemczyk ${ }^{\mathrm{E}, \mathrm{F}}$ \\ Department of Otolaryngology, Medical University of Warsaw, Poland \\ A - research concept and design; $B$ - collection and/or assembly of data; $C$ - data analysis and interpretation; \\ $D$ - writing the article; $E$ - critical revision of the article; $F$ - final approval of the article
}

Address for correspondence

Magdalena Lachowska

E-mail: mlachowska@wum.edu.pl

\section{Funding sources}

The study was supported by the grant from the Polish National Centre for Research and Development - grant NCBiR 3E147R (No. 130035 06).

Conflict of interest

None declared

Received on June 8, 2016

Reviewed on January 8, 2017

Accepted on August 11, 2017

Published online on July 30, 2018

\begin{abstract}
Background. Among the elderly, instability leading to falls (and consequences of them) is one of the most important problems. The etiology of falls is usually complex, but balance, posture and gait problems are considered to be the most important risk factors.

Objectives. The objective of this study was to assess the usefulness of the Sensory Organization Test (SOT) in differentiating multi-fallers from single-fallers.

Material and methods. The studied group included 92 patients aged $>60$ years with balance disorders and falls in their history. The patients were divided into 2 groups: multi-fallers and single-fallers. The control group (non-fallers) included 21 individuals. The SOT was performed on the $1^{\text {st }}$ day (SOT1) and on the last day (SOT2) of rehabilitation. Mean equilibrium score (ES) of 1-3 and 4-6 sensory conditions and composite score (CS) of the SOT1 and SOT2 were analyzed. The falls were analyzed as a total number of falls while performing SOT and a number of falls in all 3 repetitions of both conditions 5 and 6 separately. In SOT conditions 1-4 there were no falls observed.
\end{abstract}

Results. The importance of SOT to differentiate fallers from non-fallers and single-fallers from multi-fallers is ambiguous.

Conclusions. The SOT may or may not indicate the differences between the groups - it does not fully explain those differences. It shows only postural dysfunction without indicating any localization in particular part of vestibular organ. The basic diagnostic evaluation in the elderly with a proneness to falls should include clinical examination and the Dix-Hallpike maneuver, supplemented with a videonystagmography (VNG), which would assess the structure of damage in vestibular organ. Posturography is of less validity in the differentiation of fallers from non-fallers.

Key words: posture, benign paroxysmal positional vertigo, gait, dizziness, inner ear

Cite as

Pierchała K, Lachowska M, Wysocki J, Morawski K, Niem czyk K. Evaluation of the Sensory Organization Test to differentiate non-fallers from single- and multi-fallers. Adv Clin Exp Med. 2019;28(1):35-43. doi:10.17219/acem/76374

DOI

10.17219/acem/76374

Copyright

Copyright by Author(s)

This is an article distributed under the terms of the

Creative Commons Attribution Non-Commercial License

(http://creativecommons.org/licenses/by-nc-nd/4.0/) 


\section{Introduction}

Among the elderly, instability leading to falls (and consequences of them) is one of the most important problems. The etiology of falls is usually complex, but balance, posture and gait problems are considered the most important risk factors. ${ }^{1,2}$ The age-dependent changes in the balance system play an important role in the incidence of dizziness in the elderly; they may be caused by pathological processes. ${ }^{3}$ Various pathological processes cause damage to the vestibular organ at any age, both in young people and the elderly. ${ }^{4}$ Increased prevalence of risk factors that cause damage to the vestibular organ in the elderly may result from a greater vulnerability of the ageing vestibular system, or from a more extended time of exposure to them in younger people. ${ }^{3,4}$ Indeed, as dizziness is reported by about $20-30 \%$ of the general population,the same ailment is reported by $40 \%$ of men and $60 \%$ of women at the age of over 70 years. ${ }^{5,6}$

Among people over the age of 65 years that fall, $15-23 \%$ report an episode of vertigo as the cause of the collapse. ${ }^{6}$ The relationship between falls and vestibular system dysfunction, however, is ambiguous. The factors that lead to its complexity are: a variety of dizziness descriptions reported by patients, the extent of damage in the vestibular system and vestibular compensation. ${ }^{7}$ Damage to the upper part of the labyrinth (e.g., after previous vestibular neuritis), manifested by weakness or absence of a response in caloric test, initiates the process of degeneration of otolithic organs. It leads to canalo- or cupulolithiasis, clinically manifested as benign paroxysmal positional vertigo (BPPV), which is the most common type of vestibular ailment. ${ }^{8}$ It may occur at any age, but its incidence in the elderly population is many times greater than in younger patients,and the peak of those incidences is between the $5^{\text {th }}$ and $7^{\text {th }}$ decade of life. ${ }^{8,9}$

Benign paroxysmal positional vertigo can be idiopathic or secondary to changes in the inner ear and/or the central nervous system (CNS), and may, therefore, be present independently, or as a consequence of the inner ear and/or CNS diseases. ${ }^{10}$ In addition to typical dizziness when changing positions, which may persist for up to 12 weeks, positional vertigo can cause long-lasting instability in about 50\% of individuals,or in severe cases, hypersensitivity to almost any head movement. ${ }^{8-11}$ Considering that the recurrences of BPPV concern about $50-55 \%$ of patients, with a recurrence rate of approx. $15 \%$ per year, it seems likely that more than half of the patients with BPPV experience prolonged instability even for many months. ${ }^{8,12}$ Thus, it seems that BPPV and consequent instability, as an independent pathology or an effect of previous pathological processes in the vestibular system (that intensifies even age-related postural control disorders), should be treated as the most important cause of imbalance and tendency to falls among the elderly. ${ }^{10,11}$

An important component of therapy and strategies of preventing falls in patients with vestibular pathology is the vestibular rehabilitation. Its beneficial effect has been shown also in the age-related imbalance. ${ }^{13}$ While effectiveness of reposition maneuvers in the treatment of BPPV is undeniable, the postural training is recommended in the treatment of instability. ${ }^{14}$ In older patients, with a variety of different health problems, postural rehabilitation improves the maintenance of balance in more than $60 \%$ of them, decreasing the intensity of symptoms and reducing disability in everyday life. ${ }^{15}$

One of the methods of rehabilitation is postural training, based on sensory conflicts and conducted on the basis of computer dynamic posturography (CDP) described by Nashner. ${ }^{16}$ Computer dynamic posturography is recognized as a useful diagnostic method, evaluating the sensory and motor skills as well as biomechanical aspects of balance. Sensory conflicts used in CDP rehabilitation are similar to those in Sensory Organization Test (SOT), the most important test in CDP. The SOT indicates the nature of the deficit in the balance system. It is a commonly used test to facilitate the selection of the proper vestibular rehabilitation method and to assess its effectiveness. The 6 SOT conditions that progress from the most stable (eyes open and solid stable platform) to the least stable (sway-referenced platform and visual screen) allow for the detection of body sway. With the ability to test the patient under varying sensory conditions, SOT allows the assessment of a patient's ability to use visual, vestibular and proprioceptive information, and to suppress incorrect information in order to maintain static balance. ${ }^{17}$ The SOT is an method often used for assessing the efficiency of postural control in different groups of pathology, as well as the assessment of the effectiveness of rehabilitation carried out by different methods. ${ }^{18-21}$

In its assumptions, rehabilitation based on sensory conflicts strengthens and accelerates the integration processes in the CNS. ${ }^{15} \mathrm{~A}$ patient learns to suppress irrelevant or erroneous information coming from dysfunctional channels and to focus on stimuli that come from an undisturbed channel. ${ }^{22,23}$ Conducting exercises in varying sensory conditions (e.g., unstable platform, altered visual stimuli) forces a patient to select the sensory information that is necessary for the maintenance of static equilibrium, and to generate appropriate motor responses. The possibility to gradually increase the difficulty of exercises puts the patient's balance against growing challenges and serves to intensify the learning process. Computer-navigated rehabilitation program provides visual feedback in real time, which makes it easier for a patient to understand the essence of the exercises, as well as to control and correct their performance, and creates the favorable conditions for "motor learning." In addition, it offers the medical personnel a possibility of supervision and constant monitoring of the treatment progress. ${ }^{24}$

The aim of the study was to assess the usefulness of the SOT as a method that differentiates multi-fallers from single-fallers. The additional aim was to evaluate the effectiveness of rehabilitation based on sensory conflicts in the studied populations. 


\section{Material and methods}

As observed in everyday clinical practice and as described in the literature, healthy elderly individuals fall, patients with vestibular pathology fall and patients with balance problems coming from nonvestibular pathology also fall. ${ }^{3,4,10-12}$ Therefore, in our material, the fallers group included patients who presented imbalance and falls in their medical history, regardless of etiology. The control group consisted of healthy individuals who described the state of their balance as appropriate to their age and without any falls. All subjects included in the study were people living in their own homes, and independent in basic life and social activities.

The studied group included 92 patients aged over 60 years who were referred by their family doctors, otolaryngologists or neurologists to our otoneurological outpatient unit due to balance disorders and falls in their medical history. Based on the number of falls during the last year, the patients were divided into the 2 following groups: multi-fallers (Multi-F, $n=47$ ) with a mean age of 73.6 years (min 60, max 80; standard deviation (SD) 6.3), and single-fallers (Single-F, $\mathrm{n}=45$ ) with a mean age of 67.5 years ( $\min 60$, $\max 80 ;$ SD 5.9). The Single-F group included patients with 1 fall in the medical history and the Multi-F group included those with 2 or more falls in their history, but none of them were subjected to vestibular rehabilitation before this study. In both groups, some patients reported episodes of acute vertigo in the past over a period of at least 6 months before the beginning of this study. Outside the mentioned conditions, each patient from both groups had to meet the following additional criteria for inclusion in the study: the ability to move and the lack of serious conditions in the locomotor system, as well as no serious conditions in the circulatory system, respiratory system or CNS that could interfere with everyday rehabilitation training program.

The control group (Non-F) included 21 individuals (mean age: 67 years; min 60; $\max 80$; SD 5.8) considered healthy from an otoneurological point of view. They did not report imbalance or falls in their history. They were volunteers interested in their state of health and were recruited from retiree clubs operating within our city.

In each patient, a clinical otoneurological evaluation was performed, including the Dix-Hallpike maneuver. The presence of typical nystagmus and/or a sensation of vertigo, even without coexisting nystagmus, were considered as an abnormal test result. After the Hallpike maneuver yielded a positive test result, the Semont maneuver was performed and the patient was scheduled for the next visit after a week; then again the diagnostic Hallpike maneuver was performed. After finding the Hallpike maneuver yielded a negative result in the next visit or a negative test result in the $1^{\text {st }}$ visit, the patient was scheduled for the essential tests of the study that started another week later. In the case of a positive Hallpike maneuver test result in the $2^{\text {nd }}$ visit, the whole procedure was repeated. The principle was that the main research project started 2 weeks after the Semont maneuver and 1 week after a negative Hallpike maneuver test result.

A videonystagmography (VNG) using Micromedical system (Micromedical Technologies Inc., Chatham, USA) was performed to assess the location of the damage in the vestibular system. The applied evaluation protocol was the one routinely used in our neurotological lab and included the following: spontaneous, gaze, positional, and positioning nystagmus test, saccade test, smooth pursuit eye movements test, optokinetic test, and caloric testing. To avoid the impact of VNG on CDP results, the VNG test was carried out 2 days before the start of the essential study, and after the preliminary SOT examination (SOT0).

The SOT was conducted using a computer program that is part of the Smart Equitest system (NeuroCom International Inc., Clacamas, USA). It was performed on the $1^{\text {st }}$ day (SOT1) and on the last day (SOT2) of rehabilitation. The SOT and vestibular rehabilitation using sensory conflicts are very similar. In order to eliminate SOT learning effect that might influence the effectiveness of rehabilitation, we conducted a preliminary SOT 2 days before starting rehabilitation and those results were not analyzed.

The SOT included 6 sensory conditions. The $1^{\text {st }} 3$ conditions (1-3) (3 trials per condition (Cond.)) were performed on a stable platform and included: Cond. 1: stable platform, eyes open, stable visual surrounding; Cond. 2: stable platform, eyes closed; Cond. 3: stable platform, eyes open, moving visual surrounding with the subject's anterior-posterior sway. The next 4-6 conditions ( 3 trials per condition) were performed on a moving platform, with the range of its motion dependent on the patient's sway: Cond. 4: moving platform, eyes open; Cond. 5: moving platform, eyes closed; Cond. 6: moving platform, eyes open, moving visual surrounding dependent on the patient's sway. During the examination patients were protected against falling with special safety harness.

Analyzed parameters of the SOT were:

- mean equilibrium score (mean ES), which is the average score of the 3 trials in each of the 6 sensory conditions. Equilibrium score is based on the assumption that the swing of a healthy person in the anterior-posterior plane does not exceed $12.5^{\circ}$. The result of balance for each trial is calculated separately by comparing the angular difference between the maximum sways of the patients and the sways of a healthy person. The result is presented as a reversed percentage between 0 and 100 - the result close to 0 indicates the limits of postural stability and the result close to 100 means almost complete stability that theoretically indicates no anteriorposterior excursion;

- composite score (CS) is a computer-generated score of the 6 SOT conditions; 
- loss of balance (LOB) in all 3 trials of each sensory condition (18 trials).

Mean ES of 1-3 and 4-6 sensory conditions and CS of the SOT1 and SOT2 were analyzed in all 3 studied groups (Single-F, Multi-F and Non-F). The falls (LOB) were analyzed as a total number of falls while performing SOT and a number of falls in all 3 repetitions of both Cond. 5 and 6 separately. No falls were observed in SOT conditions 1-4.

Vestibular rehabilitation based on the CDP was conducted using a computer program Smart Equitest System (NeuroCom International Inc.). The rehabilitation was based on daily 30-min sessions performed on weekdays for 2 weeks (a total of 10 sessions for each patient). Patients were protected against falling with a special safety harness. The rehabilitation protocol was the same for all patients with the difficulty of the task gradually increasing over time. Each rehabilitation session included 1-min exercises. The degree of exercise difficulty started from the easiest (20\%) and increased gradually to $100 \%$ with $20 \%$ steps for each next exercise. It was repeated in the described manner during every rehabilitation session for the entire period of the treatment. To assess the rehabilitation effect in each group, SOT1 and SOT2 were compared. To evaluate differences between the groups, the difference between SOT2 and SOT1 in each group was calculated (SOT2-SOT1) and then compared between groups.

The project was approved by the local Ethics Committee Review Board.

Statistical analysis of SOT data was performed using STATISTICA v. 10 software (StatSoft Inc., Tulsa, USA). The data were tested for normality, parametric and nonparametric criteria. To analyze the data, the following tests were used: Kruskal-Wallis test, Pearson's $X^{2}$ test and Wilcoxon test. A p-value $<0.05$ was considered statistically significant.

\section{Results}

\section{Sensory Organization Test 1 results}

Significant differences were found in Cond. 1-3, Cond. $4-6$ and CS between Non-F and Multi-F groups $(\mathrm{p}<0.05)$, and between Non-F and Single-F groups $(\mathrm{p}<0.05)$ (Table 1$)$. The results between Single-F and Multi-F groups were similar in Cond. 1-3 and Cond. 4-6 ( $p>0.05$ ); only CS results were found to be significantly different $(\mathrm{p}<0.05)$.

\section{Rehabilitation effect within every group}

The rehabilitation effect showed a significant improvement in Cond. 4-6 and in CS in all analyzed groups $(\mathrm{p}<0.05)$ (Table 2). Rehabilitation effect did not significantly change the results in Cond. 1-3 in all analyzed groups $(\mathrm{p}>0.05)$.

Table 2. Rehabilitation effect within every group (Non-F, Single-F and Multi-F) in SOT Cond. 1-3, 4-6 and CS

\begin{tabular}{|l|l|l|l|}
\hline \multirow{2}{*}{\multicolumn{1}{|c|}{ Analyzed parameter }} & \multicolumn{3}{c|}{ Wilcoxon test ( $p$-value) } \\
\cline { 2 - 4 } & Non-F & Single-F & Multi-F \\
\hline Rehab. effect SOT Cond. 1-3 & 0.0962 & 0.0784 & 0.7613 \\
\hline Rehab. effect SOT Cond. 4-6 & $0.0030^{*}$ & $0.0000^{*}$ & $0.0000^{*}$ \\
\hline Rehab. effect SOT CS & $0.0021^{*}$ & $0.0000^{*}$ & $0.0000^{*}$ \\
\hline
\end{tabular}

Single-F - single-fallers; Multi-F - multi-fallers; Non-F - non-fallers; SOT - Sensory Organization Test; Rehab. - rehabilitation;

Cond. - condition; CS - composite score; * significant difference $(p<0.05)$.

\section{Sensory Organization Test 2 results}

Significant differences were found in Cond. 1-3, Cond. 4-6 and in CS between Non-F and Multi-F groups $(\mathrm{p}<0.05)$ and between Single-F and Multi-F groups ( $<<0.05)$ (Table 1). Between Non-F and Single-F groups the differences were significant in Cond. $1-3$ ( $\mathrm{p}<0.05)$. The results in Cond. 4-6 and CS between those 2 groups were minor.

Table 1. Differences between all analyzed groups (Single-F, Multi-F and Non-F) in SOT1, SOT2 and rehabilitation effect

\begin{tabular}{|c|c|c|c|c|}
\hline \multirow[b]{2}{*}{ Analyzed parameter } & \multirow{2}{*}{$\begin{array}{c}\text { Kruskal-Wallis test ( } \mathrm{p} \text {-value) } \\
\text { Non-F vs Single-F } \\
\text { vs Multi-F }\end{array}$} & \multicolumn{3}{|c|}{ Mann-Whitney U test ( $\mathrm{p}$-value) } \\
\hline & & $\begin{array}{c}\text { Non-F } \\
\text { vs Single-F }\end{array}$ & $\begin{array}{c}\text { Non-F } \\
\text { vs Multi-F }\end{array}$ & $\begin{array}{l}\text { Single-F } \\
\text { vs Multi-F }\end{array}$ \\
\hline SOT1 Cond. 1-3 & $0.0022^{*}$ & $0.0013^{*}$ & $0.0018^{*}$ & 0.5739 \\
\hline SOT1 Cond. 4-6 & $0.0000^{*}$ & $0.0000^{*}$ & $0.0000^{*}$ & 0.0552 \\
\hline SOT1 CS & $0.0000^{*}$ & $0.0000^{*}$ & $0.0000^{*}$ & $0.0439^{*}$ \\
\hline SOT2 Cond. 1-3 & $0.0000^{*}$ & $0.0116^{*}$ & $0.0000^{*}$ & $0.0072^{*}$ \\
\hline SOT2 Cond. 4-6 & $0.0000^{*}$ & 0.1335 & $0.0000^{*}$ & $0.0016^{*}$ \\
\hline SOT2 CS & $0.0000^{*}$ & 0.0831 & $0.0000^{*}$ & $0.0010^{*}$ \\
\hline Rehab. effect Cond. 1-3 & 0.7161 & 0.9814 & 0.5645 & 0.4559 \\
\hline Rehab. effect Cond. 4-6 & $0.0402^{*}$ & $0.0075^{*}$ & 0.0607 & 0.8089 \\
\hline Rehab. effect CS & 0.1498 & $0.0467^{*}$ & 0.1413 & 0.7471 \\
\hline
\end{tabular}

Single-F - single-fallers; Multi-F - multi-fallers; Non-F - non-fallers; SOT - Sensory Organization Test; Rehab. - rehabilitation; Cond. - condition; CS - composite score; ${ }^{*}$ significant difference $(p<0.05)$. 


\section{Number of falls (LOB) in SOT1 and SOT2}

The total number of falls (LOB) in SOT1 Cond. 5 and Cond. 6 as well as the number of falls in all 3 repetitions of each of those SOT1 conditions were significantly different between Non-F and Multi-F groups ( $\mathrm{p}<0.05)$, and between Non-F and Single-F groups ( $<<0.05)$. No such differences were found between Single-F and Multi-F groups (Table 3).

The number of falls was significantly reduced after rehabilitation in both SOT2 conditions (Cond. 5 and 6) in the Single-F group ( $<0.05)$, and in Cond. 6 in Multi-F group $(\mathrm{p}<0.05)$.

Only in SOT2 Cond. 5 LOB results (number of falls) between Single-F and Multi-F groups were different

Table 3. Differences between all analyzed groups (Single-F, Multi-F and Non-F) in number of falls and nystagmus

\begin{tabular}{|l|c|c|c|}
\multirow{2}{*}{\multicolumn{1}{c|}{ Analyzed parameter }} & \multicolumn{3}{|c|}{ Pearson's $X^{2}$ test ( $p$-value) } \\
\cline { 2 - 4 } & $\begin{array}{c}\text { Non-F } \\
\text { vs Single-F }\end{array}$ & $\begin{array}{c}\text { Non-F } \\
\text { vs Multi-F }\end{array}$ & $\begin{array}{c}\text { Single-F } \\
\text { vs Multi-F }\end{array}$ \\
\hline LOB in SOT1 Cond. 5 & $0.0435^{*}$ & $0.0004^{*}$ & 0.1095 \\
\hline LOB in SOT1 Cond. 6 & $0.0120^{*}$ & $0.0002^{*}$ & 0.3669 \\
\hline LOB in SOT2 Cond. 5 & 0.5067 & 0.1214 & $0.0311^{*}$ \\
\hline LOB in SOT2 Cond. 6 & 0.4985 & 0.1660 & 0.4246 \\
\hline Gaze nystagmus & 0.4912 & 0.2363 & 0.3280 \\
\hline Spontaneous nystagmus & $0.0274^{*}$ & 0.2299 & $0.0029^{*}$ \\
\hline Positional nystagmus & 0.0747 & $0.0004^{*}$ & $0.0427^{*}$ \\
\hline
\end{tabular}

Single-F - single-fallers; Multi-F - multi-fallers; Non-F - non-fallers; SOT - Sensory Organization Test; LOB - loss of balance; Cond. - condition; * significant difference $(p<0.05)$.
( $<<0.05)$. In all other results (number of falls) of Cond. 5 and Cond. 6 there were minor differences between all groups (Table 3 ).

\section{Differences in rehabilitation effect between the groups}

There were no significant differences between Non-F and Multi-F groups, nor between Single-F and Multi-F groups. Significant differences were found only in Cond. 4-6 and in CS between Non-F and Single-F groups $(\mathrm{p}<0.05)$ (Table 1$)$.

\section{Structure of the vestibular organ damage}

The incidence of a spontaneous peripheral and central gaze, as well as positional and positioning nystagmus (PPN) is presented in Table 4. The major differences were found between Non-F and Single-F groups in spontaneous nystagmus ( $\mathrm{p}<0.05)$, between Non-F and Multi-F groups in positional nystagmus $(\mathrm{p}<0.005)$ and in both those nystagmus types between Single-F and Multi-F groups ( $\mathrm{p}<0.005$ and $\mathrm{p}<0.05$, respectively) (Table 3 ).

\section{Location of damage in the vestibular organ}

In Single-F group, 34 patients were diagnosed with peripheral deficits, 2 patients with central deficits and 9 subjects presented normal VNG test results. In Multi-F

Table 4. Structure of the vestibular organ damage in Non-F, Single-F and Multi-F groups (number of patients) - types of nystagmus observed in analyzed patients

\begin{tabular}{|c|c|c|c|c|c|c|c|c|}
\hline \multirow[b]{2}{*}{$\begin{array}{c}\text { Group } \\
\text { of patients }\end{array}$} & \multicolumn{2}{|c|}{ Gaze } & \multicolumn{3}{|c|}{ Spontaneous } & \multicolumn{3}{|c|}{ Positional } \\
\hline & no & yes & no & $\begin{array}{c}\text { yes } \\
\text { (peripheral) }\end{array}$ & $\begin{array}{c}\text { yes } \\
\text { (central) }\end{array}$ & no & $\begin{array}{c}\text { yes } \\
\text { (peripheral: Nylen type } \\
\text { II or PPN) }\end{array}$ & $\begin{array}{c}\text { yes } \\
\text { (central: } \\
\text { Nylen type I or vertical) }\end{array}$ \\
\hline Non-F & 21 & - & - & - & - & 14 & $\begin{array}{c}7 \\
\text { (Nylen II -1; } \\
\text { PPN - 6) }\end{array}$ & - \\
\hline Single-F & 44 & 1 & 36 & 9 & - & 17 & $\begin{array}{l}26 \\
\text { (Nylen II - 15; } \\
\text { PPN - 11) }\end{array}$ & $\begin{array}{c}2 \\
\text { (Nylen I - 2) }\end{array}$ \\
\hline Multi-F & 44 & 3 & 41 & 1 & 5 & 9 & $\begin{array}{c}30 \\
\text { (Nylen II - 7; } \\
\text { PPN - 23) }\end{array}$ & $\begin{array}{c}8 \\
\text { (Nylen I - 14; } \\
\text { Vertical - 4) }\end{array}$ \\
\hline
\end{tabular}

Single-F - single-fallers; Multi-F - multi fallers; Non-F - non-fallers; PPN - positional and positioning nystagmus.

Table 5. Location of damage in the vestibular organ in Single-F and Multi-F groups (number of patients)

\begin{tabular}{|l|c|c|c|c|c|c|}
\multirow{2}{*}{$\begin{array}{c}\text { Group } \\
\text { of patients }\end{array}$} & \multicolumn{5}{|c|}{ Peripheral } & \multicolumn{2}{c|}{ Mixed } \\
\cline { 2 - 8 } & not compensated & compensated & $\begin{array}{c}\text { compensated } \\
+ \text { PPN }\end{array}$ & $\begin{array}{c}\text { normal } \\
+ \text { PPN }\end{array}$ & $\begin{array}{c}\text { central } \\
+ \text { peripheral }\end{array}$ & $\begin{array}{c}\text { central } \\
+ \text { PPN }\end{array}$ \\
\hline Single-F & 15 & 8 & 6 & 5 & - & - \\
Central & $\begin{array}{c}\text { Normal } \\
\text { Multi-F }\end{array}$ & 7 & 7 & 9 & 12 & 2 \\
\hline
\end{tabular}

Single-F - single-fallers; Multi-F - multi-fallers; PPN - positional and positioning nystagmus. 
group, 35 patients were diagnosed with peripheral deficits, 2 patients with central deficits, 4 with mixed deficits, and 4 subjects presented normal VNG test results (Table 5).

\section{Discussion}

Our results indicated that neither the balance results in Cond. 1-3 and Cond. 4-6, nor the number of falls in Cond. 5 and 6 differentiated Single-F group from Multi-F group. Only CS results were significantly different between those 2 groups. However, the SOT did differentiate Non-F group from the other 2 groups (fallers groups). Whitney et al. also found no significant differences in CS between the Single-F and Multi-F results, but the results between Non-F and Single-Multi-F groups were also significantly different. ${ }^{25}$ However, in their study, Non-F were a non-falling group with vestibular pathology present. In our study, Non-F group consisted of healthy individuals (seniors) with no report of falls and no balance problems in their history. Contrary to Whitney et al., in our study, neither the total number of LOB in SOT1 Cond. 5 and Cond. 6, nor the a number of LOB in all 3 repetitions of each of those SOT1 conditions differentiated Single-F group from Multi-F group. ${ }^{25}$ The cause might be probably the similar structure of damage in the vestibular organ in both groups (peripheral damage in $75 \%$ of Single-F patients and in $74 \%$ of Multi-F patients), despite the significant age difference between them. Wallmann showed significant differences in CS between fallers and non-fallers. ${ }^{26}$ However, those authors did not disclose the structure of the groups: how many individuals suffered from peripheral vestibular organ damage, and how many from central vestibular organ damage, which could be the basis to explain the differences between the groups.

Butaois et al. performed the SOT in a group of healthy people over the age of 65 years and divided them into 3 groups: Non-F, Single-F and Multi-F. ${ }^{27}$ These groups were similar in age. The authors found worse outcomes of Cond. 2 and $\mathrm{R}^{\text {som }}$ (patient's ability to use inputs from somatosensory system to maintain balance; it is determined by dividing performance in Cond. 2 by performance in Cond. 1) results in Multi-F group than in Single-F and Non-F groups, and the factor that differentiated Single-F and Non-F groups was the number of LOB in the $3^{\text {rd }}$ repetition of Cond. 6. Girardi et al. in their study did not show any significance of the SOT as a method for differentiating fallers from non-fallers among vestibular pathology patients. ${ }^{28}$ However, they conducted their research on the Balance Track System that contains only 4 out of 6 SOT conditions. The authors demonstrated that the test with the most prognostic importance was limits of stability (LOS) test rather than the SOT.

Baloh et al. evaluated the sway velocity using Chattecx Balance System in the elderly with and without balance problems. ${ }^{29}$ They found a significantly greater sway velocity in patients with impaired balance than in the control group, and in those who presented the fear of falling, compared to those who did not reported such fears. However, the authors did not find any major differences in tests results neither between fallers and non-fallers, nor among those who fell as a result of loss of balance, those falling as a result of tripping or slipping and those who did not fall at all.

In our study, we expected the results between Non-F and Multi-F groups to be different. We did not expect relevant or significant differences between Non-F and Single-F groups, given that the Single-F group consisted mostly of patients with peripheral vestibular pathology (only 2 patients were diagnosed with central deficit), that the in those patients loses its vestibular pattern after a couple of weeks and that both groups were similar in age.$^{30}$ In Non-F group, 6 cases of PPN and 1 case of Nylen type II nystagmus were found; however, those subjects did not report any balance disorders or dizziness. This may suggest that even among elderly healthy, asymptomatic individuals, the VNG tests show some symptoms of disorders in the vestibular system. On the other hand, this confirms the opinion of Norre et al. that some people with pure PPN can present a normal posturographic test result. ${ }^{31}$

In our study, statistically significant differences in the SOT results between Non-F group and both groups of fallers, and no significant differences between Single-F and Multi-F groups occurred probably due to the structure of the 2 groups and the type of damage in the vestibular organ. As mentioned before, patients with peripheral impairment of vestibular organ formed a dominant and almost equal number in both groups of fallers. In addition, both groups presented similar number of patients with positional nystagmus typical for peripheral damage.

Due to a different compensation pattern of vestibuloocular reflex (VOR) and vestibulo-spinal reflex (VSR) that progresses in different ways, even in the case of compensated peripheral deficit, the vestibulo-spinal compensation might not take place. ${ }^{7}$ Therefore, the patients with peripheral deficit may present instability, independently of the level of VOR compensation. ${ }^{31}$ Despite the opinion that patients with a central vestibular deficit show more abnormal gait than patients with peripheral damage, our observations indicate that patients with central damage often present less instability than patients with uncompensated peripheral damage, as shown in our previous study. ${ }^{32,33}$ Despite the fact that the SOT was performed at least 2 weeks after the Semont maneuver, another reason for the increased body sway while standing on stable platform among our study of both fallers groups might be BPPV. Many authors have demonstrated greater sway on a stable platform, both in the frontal and sagittal plane, in patients with BPPV than in the healthy subjects, along with long-term instability, even after resolution of the typical positional vertigo. ${ }^{11,34}$ Giacomini et al. demonstrated that Epley maneuver causes the normalization of body sway only in the frontal plane, whereas in the sagittal plane, 
body sway did not undergo normalization until a period of 12 weeks. ${ }^{11}$ Stambolieva and Angov found that patients with a short history of BPPV presented visual dependence, which in these cases has been the source of acute sensory conflict, causing a significant increase in body sway with eyes open, which was not noted among patients with long duration of BPPV. ${ }^{34}$ The authors also showed that the duration of symptoms also determines the effect of reposition maneuver. In patients with a short history of BPPV, the reposition maneuver caused a significant decrease in body sway in both planes while standing on a platform with eyes open. However, with eyes closed, the decrease was observed only in frontal plane.

Theories about the mechanism of long-term instability after the reposition maneuver are varied. Giacomini et al. showed that the presence of otoliths within the posterior semi-circular canal causes disorders similar to proprioceptive ones, which temporarily alter the VSR, resulting in long-term increase of body sway in the sagittal plane. ${ }^{11}$ Stambolieva and Angov did not exclude the presence of residual otoliths that modified the dynamics of an affected semicircular canal, but the authors' study about smaller spectral density in the low frequency range of the spectrum confirmed the hypothesis that the alteration of the otolithic functions might be due to some unequal loading of the utricular macula. ${ }^{34,35}$ This means that as long as the Epley maneuver causes only the resolution of typical vertigo and decrease of body sway in the frontal plane, the resolution of long-term instability as a consequence of impaired dynamics in the semi-circular canal and damaged utricle requires stimulation of other sensory systems and central postural adaptation mechanisms. Thus, those patients after the Epley maneuver should be referred to postural training. ${ }^{14}$

In our study, rehabilitation based on sensory conflicts significantly improved results in SOT2 Cond. 4-6 and CS in all studied groups. In SOT2 Cond. 1-3, there was no substantial improvement. Significant differences were found between Non-F and Multi-F groups and between Single-F and Multi-F groups in all SOT2 conditions and in CS. It is interesting that the significant differences in SOT2 between Non-F and Single-F groups concerned only the equilibrium results in Cond. $1-3$, probably because both groups improved results in these conditions by almost the same amount, which still makes the results significantly different between the 2 groups, as in the SOT1. The lack of significant differences between Non-F and Single-F groups in SOT2 Cond. 4-6 and in CS indicates that patients in Single-F group improved their SOT performance in Cond. 4-6 due to rehabilitation and approached the results of Non-F group, which contributed to the improvement of the overall result of the CS and, therefore, no significant differences in these parameters (SOT2 Cond. 4-6 and CS) between these groups were found.

It appears that the significant differences in all SOT2 conditions and in CS between Single-F and Multi-F groups might be due to several factors: 1) a significant difference in age between the 2 groups; 2) more patients with BPPV in Multi-F group, which does not diminish easily with short time rehabilitation; and/or 3) more patients with central and mixed disorders in Multi-F group, because people with those deficits benefit less from vestibular rehabilitation than those with peripheral deficit. ${ }^{36}$ The analysis of LOB in Cond. 5 and 6 in both SOT 1 and SOT 2 confirms those assumptions. While in SOT1 the number of LOB in Cond. 5 and 6 did not differentiate those 2 groups, in SOT2 significant differences were found in Cond. 5 in all its 3 trials. This demonstrates that in Multi-F group, the patients did not present any learning effect within the trial, were not able to use residual information from the vestibular system and their adaptation process to repeated stimuli was interfered. ${ }^{37}$

The rehabilitation effect was evaluated in all studied groups. A more distinct effect was expected in Multi-F and Single-F groups than in Non-F group, due to the vestibular organ pathology documented in these groups. However, no significant differences in the effect of rehabilitation was found between Non-F and Multi-F groups in all SOT conditions and CS, which only confirms the low adaptive capacity in Multi-F group. The effect of rehabilitation between Single-F and Multi-F groups also did not differ significantly. Significantly different results, however, were found between Non-F and Single-F groups in terms of Cond. 4-6 and in CS. After an initial elimination of learning effect, patients in Single-F group achieved an improvement in Cond. 4-6 that exceeded the learning effect according to the suggestions of Ford-Smith et al., but only by 3 points; however, this improvement contributed to the improvement of the overall result of the CS. ${ }^{38}$

It is not known, however, whether the improved performance in SOT2 Cond. 4-6 and in CS in Single-F group is actually the result of rehabilitation or further learning effect. Sensory conflicts used during rehabilitation are similar to those used in the SOT. Since Wrisley et al. showed the effect of learning in SOT results in a group of young healthy people, the interpretation of SOT results, after completed rehabilitation based on sensory conflicts, must take into account the impact of learning effect on the final result of the test. ${ }^{39}$ Taking into account the large number of patients with peripheral vestibular deficit in Single-F group in our study, the mentioned better results of the SOT after rehabilitation may be partly due to the progress of compensation started by vestibular rehabilitation itself, and partly due to the further learning effect. Another important factor is forced motor activity in these patients resulting from the need to report daily for vestibular rehabilitation conducted in our department. On the other hand, the small effect of rehabilitation and the short duration of its conduct led us to the assumption that improved results of SOT2 Cond. 4-6 and CS in Single-F group might be caused mainly by the further learning effect. 
In our study, both the results of SOT1 (Cond. 1-3 and 4-6) and LOB (in Cond. 5 and 6) did not differentiate Single-F group from Multi-F group, probably due to the similarity of the structure and the contribution of peripheral vestibular deficit and/or BPPV in both groups. The factor that showed the differences between the groups was rehabilitation. It revealed different results in all SOT2 conditions, CS and change in LOB in Cond. 5, indicating lower adaptive capacity in Multi-F group than in Single-F group. This may be the result of reverse changes in the vestibular organ, depending on the age and the presence of mixed and central deficits in Multi-F group.

In conclusion, the results of this study and the results of other authors suggest that the importance of the SOT to differentiate fallers from non-fallers and single-fallers from multi-fallers is ambiguous, which results from a diversity of groups, study inclusion criteria and research methodology applied. It might be hypothesized that different results can be expected even when testing groups that have the same type of pathology (i.e., vestibular organ pathology) because it is difficult to collect patients with an identical degree and extent of damage, the same duration of symptoms and identical degree of vestibular compensation and central adaptation. The SOT may indicate the differences between the groups, but it does not fully explain those differences - it only shows postural dysfunction without indicating its localization in particular part of vestibular organ. Moreover, the principal disadvantage of the SOT and CDP is that body sway measurements are restricted to the anterior-posterior direction. This may be crucial for patients with peripheral vestibular pathology, when interpreting their tests results to distinguish between fallers and non-fallers. ${ }^{40}$ We believe that the basic standard diagnostic evaluation in the elderly with a proneness to falls should include medical history and clinical examination supplemented by the Hallpike maneuver and VNG. The SOT for the differentiation of fallers and nonfallers does not provide any valid information.

\section{References}

1. Rubenstein LZ, Josephson KR. The epidemiology of falls and syncope. Clin Geriatr Med. 2002;18(2):141-158.

2. Overstall PW, Exton-Smith AN, Imms FJ, Johnson AL. Falls in the elderly related to postural imbalance. Br Med J. 1977;1(6056):261-264.

3. Barin K, Dodson EE. Dizziness in the elderly. Otolaryngol Clin North Am. 2011;44(2):437-454.

4. Katsarkas A. Dizziness in aging: The clinical experiences. Geriatrics. 2008;63(11):18-20.

5. Yardley L, Owen N, Nazareth I, Luxon L. Prevalence and presentation of dizziness in a general practice community sample of working age people. Br J Gen Pract. 1998;48(249):1131-1135.

6. National Institute on Deafness and other Communication Disorders. National Strategic Research Plan. Balance and the Vestibular System. Washington, DC: US Department of Health and Human Services; 1993:153-196.

7. Schaefer KP, Meyers DL. Aspects of vestibular compensation in guinea pigs. In: Flohr H, Precht W, eds. Lesion-Induced Neuronal Plasticity in Sensimotor Systems. New York, NY: Springer; 1981:197-207.
8. von Brevern M, Radtke A, Lezius F, et al. Epidemiology of benign paroxysmal positional vertigo: A population study. J Neurol Neurosurg Psychiatry. 2007;78(7):710-715.

9. Bhattacharyya N, Baugh RF, Orvidas L, et al.; American Academy of Otolaryngology - Head and Neck Surgery Foundation. Clinical practice guideline: Benign paroxysmal positional vertigo. Otolaryngol Head Neck Surg. 2008;139(5 Suppl 4):47-81.

10. Parnes LS, Agrawal SK, Atlas J. Diagnosis and management of benign paroxysmal positional vertigo (BPPV). CMAJ. 2003;169(7):681-693.

11. Giacomini PG, Alessandrini M, Magrini A. Long-term postural abnormalities in benign paroxysmal positional vertigo. ORL J Otorhinolaryngol Relat Spec. 2002;64(4):237-241.

12. Brandt T, Hupert D, Hecht J, Karch C, Strupp M. Benign paroxysmal positional vertigo: A long-term follow-up (6-17 years) of 125 patients. Acta Otolaryngol. 2006;126(2):160-163.

13. Kao CL, Chen LK, Chern CM, Hsu LC, Chen CC, Hwang SJ. Rehabilitation outcome in home-based versus supervised exercise programs for chronically dizzy patients. Arch Gerontol Geriatr. 2010;51(3):264-267.

14. Han BI, Song HS, Kim JS. Vestibular rehabilitation therapy. Review of indications, mechanisms, and key exercises. J Clin Neurol. 2011;7(4): 184-196.

15. Gauchard GC, Jeandel C, Tessier A, Perrin P. Beneficial effect of proprioceptive physical activities on balance control in elderly human subjects. Neurosci Lett. 1999;273(2):81-84.

16. Nashner LM. Computerized dynamic posturography. In: Jacobson GP, Newman CW, Kartush JM, eds. Handbook of Balance Function Testing. St. Louis, MO: Mosby Year Book; 1993:280-307.

17. Nashner LM, Peters JF. Dynamic posturography in the diagnosis and management of dizziness and balance disorders. Neurol Clin. 1990; 8(2):331-349.

18. Badke MB, Miedaner JA, Shea TA, Grove CG, Pyle GM. Effects of vestibular and balance rehabilitation on sensory organization and dizziness handicap. Ann Otol Rhinol Laryngol. 2005;114(1 Pt 1):48-54.

19. Bittar RSM, de Giacomo Carneiro Barros C. Vestibular rehabilitation with biofeedback in patients with central imbalance. Braz J Otorhinolaryngol. 2011;77(3):356-361.

20. Cohen H, Kimball KT. Decreased ataxia and improved balance after vestibular rehabilitation. Otolaryngol Head Neck Surg. 2004;130(4): 418-425.

21. Rossi-Izquierdo M, Ernst A, Soto-Varela A, et al. Vibrotactile neurofeedback balance training in patients with Parkinson's disease: Reducing the number of falls. Gait Posture. 2013;37(2):195-200.

22. Bogaerts A, Verschueren S, Delecluse C, Claessens AL, Boonen S. Effects of whole body vibration training on postural control in older individuals: A 1 year randomized controlled trial. Gait Posture. 2007: 26(2):309-316.

23. Seidler RD, Martin PE. The effects of short term balance training on the postural control of older adults. Gait Posture. 1997;6(3):224-236.

24. Balance Manager Systems. Clinical Interpretation Guide 2009. Clackamas, OR: NeuroCom International Inc.; 2009.

25. Whitney SL, Merchetti GF, Schade Al. The relationship between falls history and computerized dynamic posturography in persons with balance and vestibular disorders. Arch Phys Med Rehabil. 2006;87(3):402-407.

26. Wallmann HW. Comparison of elderly nonfallers and fallers on performance measures of functional reach, sensory organization and limits of stability. J Gerontol A Biol Sci Med Sci. 2001;56(9):M580M583.

27. Butaois S, Gueguen R, Gauchard GC, Benetos A, Perrin PP. Posturography and risk of recurrent falls in healthy non-institutionalized persons aged over 65. Gerontology. 2006;52(6):345-352.

28. Girardi M, Konrad HR, Amin M, Hughes LF. Predicting fall risk in an elderly population: Computer dynamic posturography versus electonystagmography test results. Laryngoscope. 2001;111(9):1528-1532

29. Baloh RW, Jacobson KM, Enrietto JA, Corona S, Honrubia V. Balance disorders in older persons: Quantification with posturography. Otolaryngol Head Neck Surg. 1998;119(1):89-92.

30. El-Kahky AM, Kingma H, Dolmans M, de Jong I. Balance control near the limit of stability in various sensory conditions in healthy subjects and patients suffering from vertigo or balance disorders: Impact of sensory input on balance control. Acta Otolaryngol. 2000;120(4): 508-516. 
31. Norre ME, Forrez G. Posture testing (posturography) in the diagnosis of peripheral vestibular pathology. Arch Otorhinolaryngol. 1986; 243(3):186-189.

32. Furman JM, Whitney SL. Central causes of dizziness. Phys Ther. 2000; 80(2):179-187.

33. Pierchała K, Lachowska M, Morawski K, Niemczyk K. Does effect of rehabilitation based on sensory conflicts in patients with vestibular deficits exceed learning effect? NeuroRehabilitation. 2014;34(2):343-353.

34. Stambolieva K, Angov G. Postural stability in patients with different durations of benign paroxysmal positional vertigo. Eur Arch Otorhinolaryngol. 2006;263(2):118-122.

35. Nashner LM. A model describing vestibular detection of body sway motion. Acta Otolaryngol. 1971;72(6):429-436.

36. Shepard NT, Telian SA. Programmatic vestibular rehabilitation. Otolaryngol Head Neck Surg. 1995;112(1):173-182.
37. Rosengren KS, Rajendran K, Contakos J, et al. Changing control strategies during assessment using computerized dynamic posturography with older women. Gait Posture. 2007;25(2):215-221.

38. Ford-Smith ChD, Wyman JF, Elswick RK, Fernandez T, Newton RA. Test-retest reliability of the sensory organization test in noninstitutionalized older adults. Arch Phys Med Rehabil. 1995;76(1):77-81.

39. Wrisley DM, Stephens MJ, Mosley S, Wojnowski A, Duffy J, Burkard R. Learning effects of repetitive administration of the sensory organization test in healthy young adults. Arch Phys Med Rehabil. 2007;88(8): 1049-1054.

40. Allum JHJ, Adkin AL, Carpenter MG, Held-Ziółkowska M, Honneger F, Pierchała K. Trunk sway measures of postural stability during clinical balance tests: Effect of a unilateral vestibular deficit. Gait Posture. 2001;14(3);227-237. 